\title{
In memoriam Hans Ruedi Sahli
}

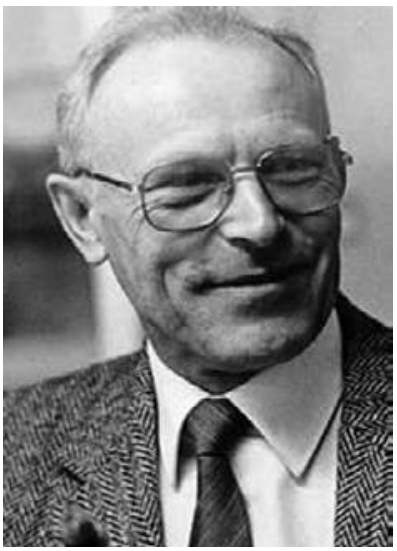

Dr. med. Hans Ruedi Sahli 1.10.1928 - 9.10.2015
«Hans Ruedi Sahli war kein Machtmensch, stellte sich nicht in den Vordergrund, sondern sah seine Arbeit darin, für die Ärzteschaft das Bestmögliche zu erreichen. Seine Voten waren klar und eindeutig. Er war auch keiner, der den Leuten nach dem Mund redete, ihnen um den Bart strich. Klar, dass einige damit so ihre Mühe hatten.»

(Zitat einer langjährigen engen Mitarbeiterin)
Der Tod von Dr. med. Hans Rudolf Sahli, FMH-Präsident von 1987 bis 1994, hat uns berührt. Doch wer war dieser Mann, der von seinen Weggefährten als so engagierter, wertvoller Mensch beschrieben wird? Als einer, der nicht an der Oberfläche hängenblieb, der gute Antennen für Menschen und Situationen hatte, als jemand, dessen bedächtige und senkrechte Art imponierte, der unprätentiös bodenständig war und dem viele seiner früheren Mitarbeitenden einiges verdanken? Wir haben uns an einem Andenken versucht, das ihm kaum gerecht wird, aber das er uns vermutlich hätte durchgehen lassen.

Hans Rudolf Sahli wurde am 1.10.1928 geboren und holte nach einer kaufmännischen Lehre in seiner Vaterstadt Bern erst die Matura nach, um nachher Medizin zu studieren. Nach Abschluss des Studiums verbrachte er einige Jahre auf der Pathologie, der Psychiatrie und in der Bernischen Höhenklinik Heiligenschwendi, bevor er sich anschliessend in sechsjähriger Weiterbildung u.a. in Basel, Solothurn und nach Aufhalten in Paris und Göteborg seinen Facharzttitel für Chirurgie erwarb.

In die FMH trat er am 1.6.1965 ein. Kurz nach seiner Praxiseröffnung im Jahr 1966 wurde er in den Vorstand des Bezirksvereins Thun und Umgebung gewählt, dessen Präsident er 1971 wurde. Von 1972 bis 1978 war er Mitglied des Bernischen Kantonalvorstands. 1978 wurde er in den Zentralvorstand gewählt und im Jahr 1984 dessen Vizepräsident.

Als er am 12. November 1987 zum Zentralpräsident der FMH gewählt wurde, war er gerade 59 Jahre alt geworden und seit 20 Jahren als freierwerbender Chirurg und Belegarzt in Thun tätig. Da nahezu gleichzeitig mit seinem Vorgänger Karl Zimmermann auch der lang- jährige Generalsekretär Hans Ott in den Ruhestand gingen, fand Hans Ruedi Sahli im Generalsekretariat nicht nur ein «abgerundetes Erbe» [1], sondern auch ein «junges Kader mit neuen Ideen und frischer Dynamik» [1] vor. Eine frühere enge Mitarbeiterin berichtete: «Das Erste, was Hans Ruedi Sahli an seinem ersten Arbeitstag zu uns Mitarbeitenden im Generelsekretariat sagte, war: 'Ich betrachte mich in diesem Haus als Gast.' Das zeigte sich in seiner Art der Zusammenarbeit mit uns: kollegial, vertrauens- und respektvoll.» Seine Amtszeit war geprägt von offener Kommunikation und engem Austausch, zum Wohle der Ärzteschaft. Gelegentlich überraschte Hans Ruedi Sahli seine Mitarbeitenden aber auch. So etwa wenn sie den Präsidenten im Keller antrafen, eigenhändig fotokopierend eine gute Zigarre rauchend.

Es liegt in der Natur der Sache, dass eine Präsidentschaft der FMH nicht ohne Konflikte verlaufen kann, und auch Hans Ruedi Sahli scheute die Auseinandersetzung nicht. Dennoch lässt es aufmerken, dass über seine Amtszeit in der Rückschau geschrieben wurde, wie «auffällig unauffällig» Dr. Sahli die Geschicke der FMH lenkte, ohne jegliche «Skandälchen und Skandale» [2].

Die Herausforderungen, denen er sich stellen musste, waren teilweise grundverschieden von den aktuellen: Die damalige «Plethora» und damit verbundene Existenzängste der Ärzteschaft sind heute schwer vorstellbar. Andere Themen sollten sich als Dauerbrenner erweisen und die von ihm eingenommene Haltung als weitsichtig. So trat er explizit für die von den damaligen «Ärzten vielfach schlecht goutierte Tarifgestaltung auf Basis betriebswirtschaftlicher Kriterien" [3] ein. Auch erkannte er die Bedeutung des hohen Orga- 
nisationsgrads der Ärzteschaft [4]. Um den Mehrwert der FMH-Mitgliedschaft zu steigern, baute er die Dienstleistungen erheblich aus v.a. über die 1978 gegründete FMH Services Genossenschaft. Ebenfalls ein prioritäres Anliegen war ihm die Qualitätssicherung, die er als "ein Gebot ärztlicher Berufsethik» [4] betrachtete. Auch hier folgten den Worten Taten und wenige Jahre später wurde die Facharztprüfung in der Weiterbildungsordnung eingeführt, gekoppelt an die - europaweit erstmalige - Einführung einer obligatorischen Fortbildung.

Besonders prägend für die Amtszeit H. R. Sahlis war jedoch die Revision der Krankenversicherung, ein Gebiet, auf dem er ein ausgesprochener Experte war. Als solcher arbeitete er auch - gemeinsam mit dem FMHGeneralsekretär F.-X. Deschenaux - in der von Ständerat Otto Schoch geleiteten Expertenkommission für die Revision der Krankenversicherung mit. Auch wenn die 1994 angenommene Totalrevision des KVG vom Entwurf dieser Kommission klar abwich, betrachtete H. R. Sahli sie doch als «die am wenigsten schlechte der heute politisch realisierbaren Alternativlösungen» [5], nicht zuletzt «aus der Erkenntnis, dass das allgemeine Interesse ständischen Eigeninteressen vorzugehen habe.» [6]

Nach zwei Amtszeiten stellte sich H. R. Sahli nicht für eine weitere Wahl zur Verfügung. Da sich sein 1994 gewählter Nachfolger Hans Heinrich Brunner aber erst deutlich später frei machen konnte, führte H. R. Sahli die Geschäfte der FMH noch bis in die ersten Monate des Jahres 1995 weiter.

Doch auch nachdem H. R. Sahli 1995 sein Amt übergeben und die Berufstätigkeit aufgegeben hatte, blieb er politisch aktiv - der leise Rückzug ins Private war nicht seine Sache. Er zog mit seiner Frau ins geliebte Val Müstair, lernte Rätoromanisch, setzte sich für den Erhalt der Mühle in Sta Maria aus dem 17. Jahrhundert ein und war massgeblich an der Verhinderung eines Heli-Flughafens im Biosphärenreservat Val Müstair beteiligt. Zudem liessen ihn noch im Ruhestand sein feines Gespür für Rechtsfragen gepaart mit seiner Hartnäckigkeit die Kostenübernahme eines Krankentransports durch die Krankenkasse ohne Anwaltsvertretung erfolgreich bis vor Bundesgericht durchfechten.

Sein Interesse am Befinden der ehemaligen ZV-Kolleginnen und -kollegen und Mitarbeiterinnen und Mitarbeiter der FMH blieb bis ins Alter lebendig, aber auch in berufspolitischen Themen blieb er auf dem Laufenden, wie sich in seinem Beitrag «Bekenntnis zur beson- nenen Re-Aktion" [7] nachlesen lässt, den er zur Jubiläumsschrift der Berner Ärztegesellschaft 2008 beisteuerte. So wie er diesen Beitrag mit einem Zitat von Wilhelm Busch schloss, bedienen auch wir uns nun - mangels treffenderer Worte - dieses Dichters: «Kein Leugnen hilft, kein Widerstreben, wir müssen sterben, weil wir leben.»

\section{Eine Begebenheit - überliefert durch H. R. Sahlis Reitlehrer}

Zu den Hobbys H. R. Sahlis gehörte auch das Reiten, zu dem er durch seine Frau, Monique Sahli, kam. Am Ende seines ersten Reitkurses sagte ihm sein Reitlehrer, er erwarte ihn zum nächsten Kurs mit einem eigenen Pferd. So würde er ihn richtig kennenlernen, da ein Pferd nach Ablauf eines Jahres das Abbild seines Gebieters sei. Trotz offener Skepsis kam H. R. zum folgenden Kurs tatsächlich mit einem eigenen Pferd.

Ein gutes Jahr darauf verunglückte er am Neujahrsmorgen allein zu Pferd im Wald: Weil das Pferd über einen verschneiten Baumstamm stolperte, fiel H. R. Sahli rücklings herunter und verletzte sich am Rücken. Das Pferd, das zunächst das Weite gesucht hatte, kehrte nach etwa zehn Minuten zurück. Der Plan Sahlis, sich mit beiden Händen am Steigbügel zu halten und schliesslich bäuchlings auf den Rücken des Pferdes zu gelangen, war gewagt: Ein junges Pferd hält beim Aufsteigen niemals still!

Aber: Das Pferd stand still und H.R. konnte sich auf dessen Rücken hieven, worauf es ihn vier Kilometer weit, ganz sachte nach Hause brachte, wo er Hilfe rufen konnte. Sein Reitlehrer erklärte ihm: «Du hast das Pferd immer respektiert, hast es zwar gefordert, warst aber immer korrekt zu ihm. Und das hat Dir das Pferd auf seine Weise zurückerstattet!» Und so hatte der Reitlehrer den Menschen Hans Ruedi kennengelernt.

\section{Dr. med. Jürg Schlup, Präsident der FMH}

\section{Literatur}

1 Sahli HR. Gruss des neugewählten Zentralpräsidenten an die Mitglieder der FMH. Schweiz Ärztezeitung. 1987;68(46):2107-8.

2 Thommen A. FMH: Wer wird Präsident? In: Ars Medici 10. 2004. S. 470

3 Sahli HR. Standespolitischer Ausblick. Schweiz Ärztezeitung. 1987;68(47):2159-60.

4 Sahli HR. Gedanken zur Qualitätssicherung. Schweiz Ärztezeitung. 1988;69(19):800-2.

5 Sahli, HR. Nach dem 4. Dezember. Schweiz Ärztezeitung. 1994;(51/52):2017-8.

6 Sahli HR. Bekenntnis zur besonnenen Re-Aktion. In: Von der Geselligkeit zur Standespolitik. 200 Jahre Medizin für die Zukunft. Ärztegesellschaft des Kantons Bern (Hrsg.) 2008; S. 191-8. 